\title{
APORTES DE LA ENFERMERÍA A LA VALORACIÓN DE LA FUNCIONALIDAD MENTAL EN REHABILITACIÓN COMUNITARIA
}

\author{
Maritza Barroso Niño1
}

\begin{abstract}
Resumen:
La rehabilitación de base comunitaria en salud mental ha hecho la diferencia en la vida de muchas personas y promete seguir aportando al mejoramiento de la salud y la calidad de vida de quienes padecen trastornos mentales. No obstante, este tipo de intervenciones aún requiere fortalecer sus instrumentos y escalas de medición, en especial, aquellos asociados a la funcionalidad mental de las personas en proceso de rehabilitación. En un esfuerzo mancomunado, la organización Asociación Coometas Campesinas y Saludarte, después de diez años continuos de trabajo en esta área, y la Fundación Universitaria Juan N. Corpas desarrollaron una investigación para atender esta necesidad, en el periodo de 2015 a 2020. La investigación produjo como resultado la Batería de Estimulación y Valoración de la Funcionalidad Mental, y también permitió identificar puntos críticos que es necesario discutir en relación con la principal clasificación de la funcionalidad y la discapacidad: Ia Clasificación Internacional de Funcionalidad de la Organización Mundial de la Salud. Este artículo presenta algunas de las críticas y reflexiones que surgieron durante la investigación. Se espera que estas permitan abrir un camino de exploración para construir la comprensión de la funcionalidad mental que integre efectivamente la perspectiva desde los cuidados de la enfermería.
\end{abstract}

Palabras clave: rehabilitación psiquiátrica, salud mental, atención de enfermería.

Historial del artículo:

Fecha de recibido: 08-09-2021 - Fecha de aceptado: 07-10-2021

(c) 2021 Fundación Universitaria Juan N. Corpas. FUJNC.

Artículo Open Access bajo la Licencia Creative Commons

Atribución-NoComercial-Compartirlgual 4.0 Internacional.

DOI: https://doi.org/10.26752/revistaparadigmassh.v3iSupl.1.606

\footnotetext{
${ }^{1}$ Enfermera y especialista en perinatología por la Universidad Nacional. Docente e investigadora en la Fundación Universitaria Juan N. Corpas y líder coordinadora en el programa Rehabilitación de base comunitaria a la persona con diagnóstico de trastorno mental (A. SALUDARTE). Bogotá, D.C., Colombia. ORCID: 0000-0001-5078-2053. Autor para correspondencia. Correo electrónico: maritza. barroso@juanncorpas.edu.co
} 


\title{
CONTRIBUTIONS TO THE ASSESSMENT OF MENTAL FUNCTIONING FROM A NURSING PERSPECTIVE IN COMMUNITY-BASED REHABILITATION IN MENTAL HEALTH
}

\begin{abstract}
:
Community-based mental health rehabilitation has made a difference in the lives of many people, and promises to continue to contribute to improving the health and quality of life of those with mental disorders. However, this type of intervention still needs to strengthen its instruments and measurement scales, especially those associated with the mental functioning of people undergoing rehabilitation. In a joint effort, the organization Asociación Coometas Campesinas and Saludarte after 10 continuous years of work in this area and the Fundación Universitaria Juan N. Corpas developed a research to address this need (2015-2020). The research produced as a result the Battery of Stimulation and Assessment of Mental Functionality, and also allowed identifying critical points that need to be discussed in relation to the main classification of functionality and disability, the International Classification of Functionality of the World Health Organization. This article presents some of the criticisms and reflections that emerged during the research. It is hoped that these will open a path of exploration to build an understanding of mental functioning that effectively integrates the nursing care perspective.
\end{abstract}

Keywords: psychiatric rehabilitation, mental health, nursing care. 


\section{Introducción}

El diseño de la Batería de Estimulación y Valoración de la Funcionalidad Mental (por brevedad, se hablará de la Batería) se basa en quince años de trabajo en el cuidado y atención de las personas con trastornos mentales; en cinco años, se sistematizaron los avances en las formas de valorar ese cuidado y en el conocimiento profundo de los estudios y apuestas que otras personas dedicadas al área han desarrollado. La Batería articula estos dos mundos, el de la práctica y la teoría, y sus instrumentos derivan de la sistematización de los procesos de cuidado a partir de las clasificaciones, manuales diagnósticos y lenguajes estandarizados en salud mental, funcionalidad, discapacidad y diagnósticos en enfermería. Dicha sistematización fue, a su vez, un proceso de análisis y teorización dirigido a comprender los desfases que se presentaban entre las necesidades de los procesos de rehabilitación de base comunitaria y lo que las clasificaciones y lenguajes estandarizados existentes permitían, en particular, la Clasificación Internacional de la Discapacidad y la Funcionalidad (CIF-OMS) (Organización Panamericana de la Salud, 2015).

En el presente artículo se hace una descripción de la Batería, que enfatiza en las relaciones que guarda con la literatura: teorías, modelos, clasificaciones, manuales, etc., en el campo de la salud mental, la rehabilitación de base comunitaria, la funcionalidad, la discapacidad y el cuidado en salud mental en enfermería. El objetivo es doble: por un lado, explicitar los compromisos teóricos que subyacen a la construcción de la Batería, al evidenciar la manera en que esta incorpora múltiples lenguajes y clasificaciones estandarizadas, y, por el otro, hacer evidente el distanciamiento de la Batería frente a estos, así como las críticas que de allí derivan.

El texto se divide en tres secciones, de la siguiente manera: en la primera sección, se plantea brevemente el contexto que da lugar a la creación de la Batería. En la segunda parte, se describen tanto la Batería como sus objetivos de aplicación, así como las relaciones que guarda cada una de sus partes con la bibliografía y marcos de referencia pertinentes; a partir de allí, se explica el distanciamiento y críticas que surgieron en relación con la CIF-OMS. En la tercera y última parte, se recogen las conclusiones generales y se esboza lo que serían los comienzos de un camino de exploración para construir una comprensión de la funcionalidad mental que integre efectivamente la perspectiva desde los cuidados de enfermería.

\section{Sección I. El contexto}

La ineficacia de la atención asilar ha llevado a los profesionales a generar diversas propuestas para la atención interdisciplinar de pacientes con trastorno mental (Bouttelet et al., 2008). Una de estas propuestas es la rehabilitación de base comunitaria en salud mental, entendida, según López y Laviana (2007), como una orientación o enfoque teórico, organizativo y funcional, alternativo a otros modelos que basan la atención en hospitales psiquiátricos, la cual se caracteriza por cuatro dimensiones básicas:

a. Un planteamiento de salud pública, basado en el compromiso de atención al conjunto de problemas de salud mental, de territorios y poblaciones concretas, así como en la utilización de criterios epidemiológicos, tanto en el enfoque poblacional general como en el establecimiento de prioridades en la atención.

b. Una filosofía de la rehabilitación que dirige las intervenciones preferentemente hacia el incremento de la autonomía personal y la funcionalidad social de usuarios y usuarias, que enfatice en sus capacidades y respete sus intereses y expectativas; esto implica una atención integral, con actividades de prevención, tratamiento, rehabilitación en sentido estricto e integración social.

c. La organización de la atención mediante una red de dispositivos sanitarios y no sanitarios, con capacidad para facilitar un conjunto amplio de prestaciones accesibles y adaptables a las necesidades individuales.

d. Un funcionamiento que se basa tanto en el trabajo en equipo, con mecanismos de coordinación que aseguren la continuidad de cuidados, como en la participación de profesionales, usuarios y usuarias, familiares y otros grupos sociales (López y Laviana, 2007, p. 92). 
En este sentido, la rehabilitación de base comunitaria en salud mental busca proveer espacios protegidos, no excluyentes, que permitan atender las necesidades de las personas usuarias y de sus familiares o redes de apoyo cercanas. Por su lado, la rehabilitación, a diferencia de la medicina curativa, tiene por objetivo contener las consecuencias del deterioro, al reducir las discapacidades y la minusvalía, así como ayudar a las personas a desarrollar habilidades y estrategias que les permitan alcanzar una existencia significativa (Haffey y Johnston, 1990). La rehabilitación no busca curar el trastorno mental, sino que las personas alcancen la recuperación.

El resultado de la rehabilitación es una función que involucra principalmente las habilidades que las personas en este proceso pueden ejercer y los recursos de apoyo que aquellas tengan en sus comunidades (Anthony, 1979, 1994; Anthony et ál., 1984; Anthony et ál., 1978; Anthony y Jansen, 1984; Anthony y Liberman, 1986; Arns \& Linney, 1995; Cohen \& Anthony, 1984; Dion \& Anthony, 1987). Así, las intervenciones de rehabilitación en salud mental de base comunitaria se enfocan fuertemente en el desarrollo de las habilidades y el aseguramiento de los recursos idóneos para cada persona de la comunidad; en consecuencia, las evaluaciones de la rehabilitación deben valorar las habilidades y los apoyos presentes y necesarios de los clientes.

La evaluación funcional de las personas es un área especialmente importante en la rehabilitación, en tanto permite obtener información sobre las falencias cruciales a intervenir, las dinámicas más adecuadas y su efectividad; sin embargo, aunque en el área de los resultados clínicos existen cientos de instrumentos para su uso con personas con trastornos de salud mental. La razón principal es que algunos instrumentos existentes se centran en el diagnóstico y los síntomas psiquiátricos; otros, en las funciones neuropsicológicas;ciertos, en el comportamiento y las habilidades;algunos en el funcionamiento y el estado, y otros en los recursos (Dahlstrom et al., 1972; Derogatis, 1994; Derogatis et ál., 1976; Eisen et ál., 1994; Honigfeld y Klett, 1964). Y sin embargo, como lo explican claramente Schaber et ál., "[e]l desafío en la evaluación cognitiva es que las herramientas de detección estandarizadas para la cognición global no se vinculan con la capacidad de realizar las actividades de la vida diaria" (Schaber, 2013). Esto resulta altamente problemático, al tener en cuenta que la funcionalidad, desde la perspectiva de la rehabilitación, está íntimamente relacionada con la habilidad efectiva para desarrollar las actividades de la vida diaria en el entorno adecuado para ello.

La carencia de herramientas de medición que integren tanto las habilidades de la persona como sus recursos actuales y necesarios, desde la perspectiva de las habilidades para realizar las actividades de la vida diaria, es especialmente problemática en el campo del cuidado de la enfermería. Dado el curso crónico de los trastornos mentales, se requiere asegurar la estabilización de síntomas para evitar el deterioro y promover calidad de vida, lo que amerita valoraciones semanales y supervisión diaria de los comportamientos predictores que orienten los cuidados o custodias en las áreas de riesgo para estados críticos. En tanto el personal de enfermería es el que tiene la relación de cuidado más cercana con las personas y presta el apoyo en el desempeño de las actividades básicas e instrumentales de la vida diaria, es crucial que aquel cuente con las capacidades y herramientas necesarias para realizar su valoración y seguimiento. Sin embargo, los instrumentos específicos de enfermería que guían la valoración de aspectos críticos de la disfunción mental son limitados y, de la misma forma, la aplicación de la taxonomía de los diagnósticos enfermeros podría convertirse en un obstáculo en la orientación del cuidado comunitario de enfermería de personas con trastornos mentales. Adicionalmente, las valoraciones funcionales son todavía muy globales, pues no penetran en el inestable mundo de la psicosis y por tanto no dan luces oportunas en las franjas de cambio de síntomas en el trastorno mental. Tal es el caso de la Escala Funcional Global o el Índice de Katz, que evalúan los cambios en el estado de salud declinante según las actividades básicas de la vida diaria; o de la escala de Lawton, que evalúa el grado de autonomía en las actividades instrumentales de la vida diaria.

Este complejo contexto motivó la realización, en 2015, de un proyecto de investigación en conjunto entre la Fundación Universitaria Juan N. 
Corpas y la Asociación Coometas Campesinas y Saludarte (A. SALUDARTE), orientada a facilitar herramientas ágiles que contribuyan a la planeación del cuidado de enfermería de la población diagnosticada con trastorno mental. El objetivo principal de la investigación era optimizar una serie de instrumentos de valoración de la funcionalidad mental en la rehabilitación de base comunitaria, para lograr integrar el lenguaje estandarizado de enfermería. La principal conclusión de la investigación es que una adecuada valoración de la funcionalidad mental en la atención comunitaria de cuidado social, que integre el lenguaje estandarizado enfermero, requiere de una batería completa de estimulación y valoración. Esta debe permitir: a) tutorar el cuidado, hacer seguimiento y evaluar el proceso de cuidado de la persona diagnosticada con trastorno mental en proceso de rehabilitación; b) valorar su funcionalidad mental, diagnosticar y planear el cuidado, y c) diseñar el aspecto terapéutico de las relaciones de cuidado. El principal resultado de la investigación fue el diseño y validación de dicha batería. El resultado secundario fue una serie de reflexiones y críticas sobre las principales clasificaciones y guías en funcionalidad y discapacidad; en este caso, el artículo está centrado específicamente en la CIF-OMS.

\section{Sección II. La Batería y su acercamiento crítico a los postulados de la CIF-OMS}

La Batería de Estimulación y Valoración de la Funcionalidad Mental consiste en un conjunto de seis instrumentos que recogen tres procesos, diseñados para que las personas del equipo de cuidado (profesionales, cuidadores, auxiliares y otros a cargo de la rehabilitación de base comunitaria) realicen: a) una adecuada estimulación de las funciones involucradas en el desarrollo de múltiples actividades de la vida diaria; b) un registro sistemático de las respuestas ante la estimulación; c) una valoración de la funcionalidad mental basada en estos registros y los perfiles de funcionalidad de las personas en rehabilitación, y d) la planeación de los cuidados, diagnósticos de enfermería, resultados e intervenciones NANDA Internacional (2008), NIC. NOC. NNN. La articulación entre las diferentes partes de la Batería permite tutorar el cuidado; planear apoyos, actividades y correctivos, y diseñar relaciones de cuidado para los sujetos individuales y colectivos que se encuentran en procesos de rehabilitación de base comunitaria.

La Batería valora diferentes dominios ${ }^{2}$ de funcionalidad en la cotidianidad, en tres áreas de intervención clave en salud mental (autocuidado, convivencia y ocupación), al relacionar las funciones mentales involucradas en la ejecución de los dominios. Múltiples estudios han demostrado que el deterioro cognitivo propio de algunos trastornos mentales se manifiesta en dificultades en el desarrollo de actividades de la vida diaria y que estas dificultades corresponden a habilidades de proceso específicas (Cooke et al., 2000). También se ha demostrado que la valoración de la funcionalidad cognitiva permite predecir los niveles esperados de ejecución funcional de actividades de la vida diaria (Schaber et al., 2013). Así pues, el enfoque de la Batería resulta ideal a la hora de abordar la funcionalidad a partir de un enfoque combinado, que cruza las funciones mentales con las actividades de la vida diaria en las que estas se involucran. Lo anterior no solo porque es más completo que hacerlo únicamente desde uno de los dos componentes, sino también porque permite explorar aspectos combinados que son altamente informativos. Adicionalmente, esto responde al gran reto detectado por Schaber et aSl. (2013), al vincular la capacidad de realizar las actividades de la vida diaria a la valoración de la cognición global. A continuación, se describen los instrumentos de la Batería de acuerdo con el proceso al que corresponden:

Parte 1. Estimulación y registro: la batería contiene tres cintas de funcionalidad mental (CFM), una por cada eje de intervención. Las CFM permiten guiar de manera sistemática la estimulación de diecinueve funciones mentales intervenidas en cuarenta y cuatro dominios, así como registrar la respuesta a la estimulación y la calidad de la ejecución. Las CFM están diseñadas para un registro de un mes calendario.

Parte 2. Semaforización de éxito: la Batería incorpora una matriz de semaforización (MS) para el registro del nivel de éxito de las veinte actividades-participaciones / dominios que hacen parte de las CFM en los ejes de intervención autocuidado y convivencia. Una vez diligenciada, la MS 
permite visualizar los resultados de la realización de las actividades tanto a nivel individual como a nivel de la comunidad, y los ubica en tres niveles: bueno, regular y malo.

Parte 3. Matriz de evolución (ME): la ME es un instrumento que integra los registros de cada categoría y arroja, de manera automática, los siguientes resultados: a) nivel del déficit del funcionamiento mental (porcentaje), de acuerdo con las bases conceptuales de la CIF - OMS; b) histórico semestral del nivel de déficit de funcionamiento mental; c) perfil de funcionalidad mental por eje de intervención (autocuidado, convivencia, ocupación); d) perfil por calidad de la ejecución, y e) perfil según calidad de la ejecución por eje de intervención. A partir de estos datos, se logran los siguientes diagnósticos: a) clasificación de la extensión del déficit (primer calificador); b) naturaleza del déficit (segundo calificador); c) perfil de desempeño menor logro; d) porcentajes de apoyo que ha definido la CIF, según el primer calificador CIF-OMS, y e) diagnósticos enfermeros. Parte 4. Plan de cuidados que parte de los diagnósticos de enfermería, intervenciones terapéuticas recomendadas o resultados esperados, con indicadores que mide la Batería, según la evidencia científica para los cuidados enfermeros (según la CIF-OMS y NANDA-NIC-NOC) que aparecen en documento anexo.

La información arrojada por la ME permite hacer una valoración integral de la funcionalidad mental de la persona cuidada e identificar las intervenciones y estimulaciones que requiere, al permitir ajustar las CFM y, así, guiar el cuidado y la estimulación de acuerdo a las necesidades específicas ya identificadas. En otras palabras, esta información es el insumo base para que el equipo defina un plan de cuidados acorde a la condición, contexto y necesidades de la persona, basado en registros que responden a la situación particular. Estos se analizan a partir de las categorías de los principales lenguajes estandarizados para la valoración de la funcionalidad y los diagnósticos e intervenciones enfermeras.

En conclusión, la Batería tiene una naturaleza adaptativa, de acuerdo con la cual la información recolectada y transformada a partir del proceso de valoración permite modificar los campos de esta (dominios, funciones mentales y funciones de ejecución), para ajustarlos a las necesidades y características específicas de cada persona cuidada. Esto permite que la Batería se acople a la naturaleza cambiante del cuidado y a su vez optimice tanto la planeación de la estimulación y el cuidado como el seguimiento de la evolución de logros. Puesto que el cuidado y el autocuidado es universal en la especie humana y comparte categorías de funcionalidad mental, como ha quedado explicado para el cuidado de personas con trastorno mental, se sugiere el uso en población general, para así potenciar la agencia de todo autocuidado y cuidado.

Utilizar los perfiles de funcionalidad que la Batería produce permite procesos de rehabilitación personalizados y con rendimientos más estimulantes, lo que aligera la pendiente de deterioro definida en la historia natural del trastorno metal. A su vez, la articulación que hace la Batería de los componentes y dominios de la CIF-OMS, con la taxonomía de las funciones mentales propuesta por Mariño y Pabón (2011), así como los diagnósticos e intervenciones NANDA-NIC-NOC, asegura la unidad en el diseño de planes, permite comparar resultados a nivel global y provee el marco para la interoperatividad con los demás servicios de atención en salud mental. Esto permite que los procesos de rehabilitación basada en comunidad, que históricamente han estado desarticulados de otras formas institucionales de intervención en salud, puedan dialogar con estas.

El proceso de diseño de la Batería se nutrió de la experiencia de quince años de trabajo en el cuidado y atención de la salud mental en A.SALUDARTE, quienes se dedican a la protección y rehabilitación de personas con diagnóstico de trastorno mental y tienen un conocimiento profundo de los estudios y apuestas que otras personas en el área han desarrollado. En este sentido, la Batería articula dos mundos: el de la práctica y la teoría. Sus instrumentos reflejan una sistematización de los procesos de cuidado desde la perspectiva de enfermería, que se nutre de las clasificaciones, manuales diagnósticos y lenguajes estandarizados en salud mental. Es importante resaltar la perspectiva de la enfermería porque su función en los procesos de cuidado es diferente del 
de otras disciplinas: este cuidado se construye en contacto permanente con las personas cuidadas, pues prestan el acompañamiento y apoyo para las actividades de la vida diaria. En este sentido, el personal de enfermería tiene acceso a todos aquellos detalles que son altamente significativos desde la perspectiva de la funcionalidad y que otros profesionales no atestiguan. Por eso, su papel tanto en los procesos de rehabilitación de base comunitaria en salud mental como en su evaluación es primordial.

El hecho de que la Batería se haya construido desde la perspectiva del cuidado de enfermería permitió evidenciar una serie de elementos problemáticos en relación con varias clasificaciones y lenguajes estandarizados que son referentes obligados dentro del universo de la atención y el cuidado de la salud mental, principalmente la CIF - OMS. A continuación, se describen las relaciones que tiene cada una de las partes de la Batería con la literatura y marcos de referencia pertinentes, y a partir de allí se explica el distanciamiento y críticas que surgieron en relación con la CIFOMS.

\section{Generalidades de la relación entre la Batería de Estimulación y Valoración de la Funcionali- dad Mental y la CIF-OMS}

Como clasificación, la CIF - OMS no establece un modelo para el "proceso" del funcionamiento y la discapacidad. Sin embargo, puede utilizarse para describir dicho proceso proporcionando los medios para delinear los diferentes "constructos" y dominios. Proporciona un abordaje, desde una perspectiva múltiple, a la clasificación del funcionamiento y la discapacidad como un proceso interactivo y evolutivo. Proporciona las "piezas de construcción" para poder crear modelos y estudiar los diferentes aspectos del proceso. En este sentido, podemos considerar la CIF - OMS como un idioma y los textos que se pueden crear dependerán de los usuarios, de su creatividad y de su orientación científica. (Organización Mundial de la Salud, 2001, p. 29)

La Batería hace uso de las piezas de construcción propuestas por la CIF-OMS, y las articula al seguir el modelo de atención de A.SALUDARTE. En primer lugar, la Batería, así como la CIF-OMS, tiene en cuenta no solo los estados de salud, sino también otros aspectos relacionados con esta y el bienestar, y aborda las deficiencias: signos y síntomas, como problemas de las funciones mentales asociados con las condiciones de salud. La propuesta teórica y clasificatoria de la CIF-OMS, tal y como la incorpora la Batería, permite remplazar la visión basada en los conceptos tradicionales de deficiencia, discapacidad y minusvalía asociados con los trastornos mentales por una visión más compleja, en la que "el funcionamiento y la discapacidad de una persona se conciben como una interacción dinámica entre los estados de salud (enfermedades, trastornos, lesiones, traumas, etc.) y los factores contextuales" (Organización Mundial de la Salud, 2001, p. 12).En segundo lugar, "incluyen la visión, audición, movilidad, aprendizaje y memoria [...][y] aquellas áreas de funcionamiento, que aun teniendo una fuerte relación con una condición de salud, no se incluyen dentro de las responsabilidades prioritarias de los sistemas de salud, sino más bien, dentro de las de otros sistemas que contribuyen al bienestar global (Organización Mundial de la Salud, 2001, p. 206) En otras palabras, en línea con la perspectiva de la rehabilitación, la Batería parte de los componentes de la salud, para abordar el estado de la persona, y no la perspectiva de las consecuencias de las enfermedades, que se refiere al efecto de las enfermedades.

\section{Los componentes de la salud}

Las cintas de funcionalidad mental (CFM), instrumentos que componen la primera parte de la Batería, guían la estimulación (tutoran en cuidado) y registro del resultado de esta en la ejecución de los dominios. En estos instrumentos se hace operativa, por primera vez, la relación conceptual entre la Batería y la CIF-OMS.

La CIF-OMS comprende cuatro componentes de la salud, distribuidos de la siguiente forma. Los componentes de funcionamiento y la discapacidad, que son: a) las funciones y estructuras corporales, y b) las actividades y la participación. Los componentes de factores contextuales, que son: c) los factores ambientales, y d) los factores personales. Cada componente comprende su 
propia serie de dominios, que se evalúan a partir de los constructos y su relación con los calificadores (véase la tabla 1).

En relación con el primer componente, las CFM incluyen tanto las actividades como las participaciones nombrada como dominios CIF-OMS En este sentido, los dominios de las CFM se alimentan de la lista comprensiva ofrecida por la CIFOMS (Organización Mundial de la Salud, 2001, pp. 131-171).

En relación con el otro componente, mientras que la $\mathrm{CIF}-\mathrm{OMS}^{3}$ incluye tanto las funciones corporales y mentales como las estructuras físicas/ materiales, la Batería únicamente incluye las fun- ciones mentales, lo que le permite avanzar por los linderos de la fisiología, en tanto su objeto se centra en la estimulación y registro de la funcionalidad mental, y no pretende incluir los aspectos anatómicos o neuronales asociados a estas.

Mientras que los dominios del componente de actividad y participación de las CFM siguen la lista de la CIF-OMS, las funciones mentales se elaboran a partir de trabajos en valoración de funciones mentales desde enfermería (Mariño y Pabón, 2011). Hay tres razones para esto: la primera, y más importante, es que el trabajo de Mariño y Pabón (2011) está construido desde la perspectiva del cuidado de enfermería, la cual se enfoca en la manera en que las funciones hacen

Tabla 1. Visión de conjunto de la CIF-OMS

\begin{tabular}{|c|c|c|c|c|}
\hline & \multicolumn{2}{|c|}{ Parte 1. Funcionamiento y discapacidad } & \multicolumn{2}{|c|}{ Parte 2. Factores contextuales } \\
\hline Componentes & $\begin{array}{l}\text { Funciones } \\
\text { estructurales } \\
\text { corporales }\end{array}$ & Actividades y participación & $\begin{array}{l}\text { Factores } \\
\text { ambientales }\end{array}$ & $\begin{array}{l}\text { Factores } \\
\text { personales }\end{array}$ \\
\hline Dominios & $\begin{array}{l}\text { Funciones } \\
\text { corporales } \\
\text { Estructuras } \\
\text { Corporales }\end{array}$ & $\begin{array}{c}\text { Áreas vitales } \\
\text { (tareas, acciones) }\end{array}$ & $\begin{array}{c}\text { Influencias externas } \\
\text { sobre el } \\
\text { funcionamiento } \\
\text { y la discapacidad }\end{array}$ & $\begin{array}{c}\text { Influencias internas } \\
\text { sobre el } \\
\text { funcionamiento y } \\
\text { la discapacidad }\end{array}$ \\
\hline Constructos & $\begin{array}{l}\text { Cambios en las } \\
\text { funciones } \\
\text { corporales } \\
\text { (fisiológico) } \\
\text { Cambios en las } \\
\text { funciones de } \\
\text { cuerpo } \\
\text { (anatómicos) }\end{array}$ & $\begin{array}{l}\text { Capacidad de } \\
\text { realización de tareas en } \\
\text { entorno uniforme. } \\
\text { Desempeño/realización } \\
\text { de tareas en entorno } \\
\text { real }\end{array}$ & $\begin{array}{c}\text { El efecto } \\
\text { facilitador o de } \\
\text { barrera de las } \\
\text { características del } \\
\text { mundo físico, social } \\
\text { y actitudinal }\end{array}$ & $\begin{array}{l}\text { El efecto de los } \\
\text { tributos de la } \\
\text { persona }\end{array}$ \\
\hline \multirow{2}{*}{$\begin{array}{l}\text { Aspectos } \\
\text { positivos }\end{array}$} & $\begin{array}{l}\text { Integridad } \\
\text { funcional y } \\
\text { estructural }\end{array}$ & Actividades participación & \multirow[t]{2}{*}{ Facilitadores } & \multirow[t]{2}{*}{ No aplicable } \\
\hline & \multicolumn{2}{|c|}{ Funcionamiento } & & \\
\hline \multirow[t]{2}{*}{$\begin{array}{l}\text { Aspectos } \\
\text { positivos }\end{array}$} & Deficiencia & $\begin{array}{c}\text { Limitación en } \\
\text { la actividad, restricción } \\
\text { en la participación }\end{array}$ & \multirow[t]{2}{*}{ Barreras/obstáculos } & \multirow[t]{2}{*}{ No aplicable } \\
\hline & & iscapacidad & & \\
\hline
\end{tabular}

Fuente: Organización Mundial de la Salud (2001, p. 16).

\footnotetext{
3“Actividad es la realización de una tarea o acción por una persona. Participación es el acto de involucrarse en una situación vital” (Orga-
} nización Mundial de la Salud, 2001, p. 21). 
parte de procesos globales para el desarrollo de las actividades de la vida diaria de las personas, perspectiva que, a diferencia de las más biologicistas, está en consonancia con la rehabilitación en salud mental de base comunitaria. En segundo lugar, su trabajo incluye un mayor rango de funciones que dialogan con la observación y acompañamiento de las actividades de la vida diaria. En tercer lugar, Mariño y Pabón (2011) definen con mayor claridad la relación entre los grupos funcionales, las funciones generales y las sub-funciones, lo cual permite realizar análisis más robustos en relación con los perfiles de funcionalidad.

Con respecto al componente de factores ambientales de la CIF-OMS, surge un segundo distanciamiento. Los factores ambientales son definidos por la CIF-OMS como las influencias externas sobre el funcionamiento y la discapacidad, y [...] refieren a todos los aspectos del mundo extrínseco o externo que forma el contexto de la vida de un individuo, y como tal afecta el funcionamiento de esa persona. Los factores ambientales incluyen al mundo físico natural con todas sus características, el mundo físico creado por el hombre, las demás personas con las que se establecen o asumen diferentes relaciones o papeles, las actitudes y valores, los servicios y sistemas sociales y políticos, y las reglas y leyes. (Organización Mundial de la Salud, 2011, p. 208)

La CIF-OMS recomienda hacer una medición independiente de los factores ambientales porque su objetivo es ofrecer un diagnóstico del nivel de funcionalidad y discapacidad. En efecto, si "el funcionamiento y la discapacidad de una persona se conciben como una interacción dinámica entre los estados de salud (enfermedades, trastornos, lesiones, traumas, etc.) y los factores contextuales" (Organización Mundial de la Salud, 2001, p. 12). Entonces, ambos factores contribuyen al resultado y deben medirse independientemente para ofrecer un diagnóstico.

En contraste con esto, la Batería está construida para ser aplicada en ambientes de rehabilitación basada en comunidad y para que sus mediciones contribuyan a optimizar los procesos de rehabi- litación. Es en entornos protegidos especializados en rehabilitación, es decir, que tienen una infraestructura, personal y programas diseñados para cumplir dicho objetivo, que se ajustan a las necesidades y apoyos que las personas residentes requieren para poder llevar a cabo sus actividades de la vida diaria. En este sentido, en el proceso de valorar la funcionalidad de la persona en el desarrollo de sus funciones, se identifica inmediatamente si es necesario modificar o incluir alguno de los apoyos en su entorno. En otras palabras, en tanto la valoración de la funcionalidad tiene lugar durante una relación de cuidado/ atención/apoyo, la respuesta hallada habla de la relación entera y no solo de uno de sus polos. Así, el mantenimiento del entorno protegido está a cargo de todo un equipo de cuidado especializado en procesos de rehabilitación, cuyo norte apunta a integrar en lugar de fragmentar. Es por esta razón que la Batería, a diferencia de la CIFOMS, no toma el componente de los factores ambientales por separado .

La diferencia entre la CIF-OMS y la Batería que se acaba de explicar no es superficial, de hecho, deriva de las diferencias en la orientación conceptual con la que fueron construidas ambas herramientas: mientras que en la CIF-OMS priman intereses asociados a la toma de decisiones en salud pública y por ello integra, con mayor o menor éxito, los modelos médico y social, la Batería tiene claros intereses en aportar a la rehabilitación en la salud mental de las personas en procesos comunitarios. Por esta razón, en sus CFM trae a la realidad el tipo de valoración de la funcionalidad que requieren los centros de rehabilitación basada en comunidad, espacios en los que el modelo social ya está en ejecución, aún si es de manera parcial. Es importante tener esto en cuenta porque abre una serie de preguntas acerca de la idoneidad de lo propuesto por la CIF-OMS, cuando el interés desde el cuidado social es la rehabilitación en salud mental basada en comunidad: ¿es la orientación biopsicosocial de la CIF-OMS adecuada para la valoración de la funcionalidad mental en el interior de los centros de rehabilitación de base comunitaria?, ¿es coherente con el enfoque de la rehabilitación mantener los aspectos del enfoque médico que 
la CIF-OMS mantiene? Estos aspectos se verán con más claridad en la sección sobre los calificadores en este mismo artículo.

El debate en relación con las funciones mentales tal y como se mencionó en la sección anterior, las funciones mentales empleadas en la Batería siguen la categorización de funciones mentales ofrecida por Mariño y Pabón (2011) en su Manual Valoración de Funciones Mentales. La razón principal para esto es que no todas las taxonomías de las funciones mentales son compatibles con, o favorables para, la perspectiva de la rehabilitación en salud mental basada en comunidad y, a diferencia del Manual de Mariño y Pabón (2011), no resulta claro qué tipo de taxonomía sigue la CIF-OMS.

Existe un álgido e importante debate acerca de cuáles son los criterios adecuados de clasificación de las funciones mentales; en consecuencia, tampoco existe un consenso absoluto en relación con cuáles son las principales funciones mentales, cómo se agrupan y cómo se relacionan (Lezak et ál., 2004; Mesulam, 2000). Tal y como Harvey (2019) lo explica:

Hay varias formas de conceptualizar los dominios de capacidad cognitiva. Estas incluyen la clasificación por el proceso general involucrado, como la memoria o la atención, el lenguaje o el funcionamiento ejecutivo. Otras estrategias se basan en funciones cerebrales regionales, derivadas sobre la base de estudios de lesiones, que caracterizan las funciones como originadas en el lóbulo frontal, lóbulo temporal, lóbulo parietal, hipocampo u otras estructuras. Una organización adicional de la estructura (de las funciones cognitivas) es jerárquica y se basa en la complejidad de las operaciones... [a] menudo denominada de arriba hacia abajo (top-down). (p. 227)

Y más adelante:

Aunque en general se acepta la naturaleza de la mayoría de estos dominios, existen claras inconsistencias en la literatura clínica y de investigación. La mayoría de las inconsistencias se encuentran en dominios amplios que pueden incluir procesos de múltiples componentes. Si estos procesos pertenecen a dominios más genera- les (funcionamiento ejecutivo) o un dominio más simple (velocidad de procesamiento), a menudo no está claro. (Harvey, 2019)

Originalmente, las funciones mentales fueron comprendidas en relación con su localización en las áreas del cerebro en las que parecía que ocurrían los diferentes procesos (Babcock, 1930). De hecho, esta es una perspectiva que continúa teniendo auge en la actualidad (Damasio y Damasio, 1989). Es más, el Manual Diagnóstico y Estadístico de los Trastornos Mentales (DSM5) (American Psychiatric Association, 2013) se basa en este tipo de aproximación taxonómica a las funciones mentales (Lezak et ál., 2004; Andrianopoulous et ál., 2017).

Una corriente emparentada con la anterior basa su categorización de las funciones mentales en el estudio de circuitos y se centran en la activación e interacción de estos (López-García et ál., 2016). Tal y como Grossman e Irwin (2016) lo expresan:

En lugar de localizar una deficiencia en un locus anatómico específico, nuestra perspectiva sobre las relaciones cerebro-conducta es más consistente con un enfoque que se centra en la interrupción de una red neuronal o de conexión a gran escala. En este enfoque, cada red está compuesta por múltiples nodos de materia gris que están interconectados por proyecciones dentro de la materia blanca, y estos tractos de materia blanca sirven para integrar el funcionamiento de los nodos de materia gris. (p. 385)

En contraste con las dos perspectivas mencionadas, otras personas, como Al-Aidroos et al. (2012), abordan la taxonomía de las funciones mentales a partir de perspectivas conocidas como top-down, cuya idea central "es que las operaciones sensoriales y perceptuales básicas son menos complejas y el razonamiento y la resolución de problemas, conocidos como funciones ejecutivas, son las más complejas" (Harvey, 2019, p. 227).

La CIF-OMS contiene una lista comprensiva de funciones mentales (Organización Mundial de la Salud, 2001, pp. 73-83). Esta lista no define grupos de funciones, sino que se limita a dividirlas 
entre funciones globales y funciones específicas, y no explicita qué relaciones existen entre estos grupos ni en qué tipo de taxonomía se emplea (top-down, taxonomía basada en el proceso general involucrado, en las regiones cerebrales de las funciones o en las redes de circuitos y su interacción). Estas omisiones en el texto no deben pasar desapercibidas para quienes emplean el lenguaje que ofrece la CIF-OMS para construir modelos e instrumentos. Por el contrario, es crucial saber qué compromisos teóricos hay detrás de la clasificación de funciones mentales que hace la CIF-OMS para garantizar que estas estén en consonancia con los objetivos y compromisos de la construcción expuesta.

La división ofrecida por la CIF-OMS entre funciones generales y funciones específicas no parece corresponder a la diferenciación que hacen las posturas top-down entre funciones básicas y funciones complejas, porque la CIF-OMS incluye las funciones relacionadas con la energía y los impulsos, y las funciones del sueño en el grupo de funciones generales, las cuales no clasifican como de alta complejidad en el sentido en que lo entienden los enfoques top-down. Tampoco parece coincidir con los enfoques taxonómicos que se basan en el proceso general involucrado, en tanto la atención, memoria y lenguaje aparecen clasificadas como funciones específicas y no como procesos generales, y se encuentran separadas de algunas otras funciones que contribuyen a dichos procesos; además, tampoco se tiene en cuenta la categoría de funciones ejecutivas. Dicho esto, resultaría ciertamente sorprendente que la CIF-OMS, al tratarse de un manual que busca tener una visión integral de la funcionalidad y la discapacidad, utilizara aproximaciones que se basan en la ubicación cerebral de las funciones, dada la carga biologicista de estos enfoques. No obstante, ante el silencio en relación con ello y los aspectos del modelo médico que la CIF-OMS mantiene, no es una opción que pueda ser descartada.

Al tener en cuenta la dificultad del debate en relación con la definición y delimitación de las funciones mentales y el silencio de la CIF-OMS en los compromisos teóricos que subyacen a su taxonomía, la Batería fue elaborada al seguir el enfoque propuesto por Mariño y Pabón (2011), producido en la Facultad de Enfermería de la Universidad El Bosque. Aquella es cercana en la comprensión de las pesquisas del profesional de enfermería cuando busca elaborar planes de cuidado que integran en el lenguaje estandarizado, NANDA Internacional (2008) y sus vínculos con NIC-NOC. El manual es, en palabras de las autoras, una guía para realizar de forma "[...] ordenada y completa una adecuada valoración de enfermería de las funciones mentales" (Mariño y Pabón, 2011, p. 7). Es decir que aborda la categorización de las funciones mentales desde la perspectiva del cuidado de enfermería, la cual se enfoca en la manera en que las funciones hacen parte de procesos globales para el desarrollo de las actividades de la vida diaria de las personas, perspectiva que, a diferencia de las perspectivas biologicistas, está en consonancia con la rehabilitación en salud mental de base comunitaria.

Además, el trabajo de Mariño y Pabón (2011) incluye un mayor rango de funciones que dialogan con la observación y acompañamiento de las actividades de la vida diaria y define con mayor claridad la relación entre los grupos funcionales, las funciones generales y las subfunciones, lo cual permite realizar análisis más robustos en relación con los perfiles de funcionalidad. Todo esto hace del manual de Mariño y Pabón (2011) un referente conceptual idóneo para el trabajo en rehabilitación de base comunitaria en salud mental desde la perspectiva enfermera, pues aporta a la construcción de modelos y herramientas de valoración de la funcionalidad en estos contextos.

\section{Los constructos y los calificadores}

La distancia entre la Batería y la CIF-OMS no se limita a los componentes; de hecho, se agudiza en los aspectos que tienen que ver con los constructos y los calificadores. Tal y como aparece en la CIF-OMS:

Los componentes del funcionamiento y la discapacidad pueden interpretarse mediante cuatro "constructos" diferentes pero relacionados entre sí. Estos "constructos" se hacen operativos cuando se utilizan los calificadores. Las funciones y estructuras corporales pueden interpretarse mediante cambios en los sistemas fisiológicos o en las estructuras anatómicas. El componente Actividades y Participación dispone de dos 
"constructos": capacidad y desempeño/realización. (Organización Mundial de la Salud, 2011, p. 12)

Los constructos tienen sus respectivos calificadores: deficiencias, limitaciones y restricciones, todos los cuales se miden con la norma, es decir, en términos de lo que pueden hacer personas que no padecen enfermedades, trastornos, daños o condiciones que comprometen la funcionalidad:

Las limitaciones y restricciones se evalúan contrastándolas con los estándares comúnmente aceptados en la población. Los estándares o normas frente a los que valoramos la capacidad y el desempeño/realización de un individuo son los de las personas que no padecen un estado de salud similar (enfermedad, trastorno, daño, etc.). La limitación o restricción mide la discordancia entre el desempeño/realización esperado y el observado. [...] Las deficiencias representan una desviación de la "norma" generalmente aceptada en relación al estado biomédico del cuerpo y sus funciones. (Organización Mundial de la Salud, 2001, pp. 18 y 24)

Esta perspectiva sobre los constructos y calificadores claramente sigue un modelo médico de la discapacidad y sus resultados ofrecen una lectura de esta como desviación de la norma, perspectiva que no está en consonancia con el abordaje de la rehabilitación basada en comunidad. Por esta razón, la Batería no comparte los mismos constructos y calificadores de la CIF-OMS.

La Batería cuenta con tres calificadores: a) si la función mental estimulada en la persona durante la ejecución de los dominios tuvo que ser auxiliada o no; b) la frecuencia con la que la persona inició la actividad, sostuvo la atención, finalizó la actividad y obtuvo la meta (medido en términos de una escala de Likert), y c) si la persona ejecutó con éxito los dominios valorados. Estos tres calificadores permiten separar los diferentes aspectos del uso de las funciones mentales en la ejecución de los dominios que es necesario identificar para llevar a cabo una adecuada rehabilitación. En primer lugar, permiten identificar si la persona tiene habilitada una función mental, pero no logra emplearla en la ejecución de ciertos dominios; en segundo lugar, permite apreciar individualmente las propiedades de las funciones ejecutivas en la ejecución de los dominios, y, en tercer y último lugar, permite comprender si las personas lograron ejecutar los dominios de forma exitosa, independientemente de los resultados obtenidos con los otros calificadores.

Es importante aclarar que estos tres calificadores no miden la funcionalidad o discapacidad de la persona, sino que dan una noción del nivel de ayudas requeridas para el desarrollo de sus actividades de la vida diaria, de las áreas en las que dichas ayudas deben ser enfatizadas y de la evolución de su proceso de rehabilitación. Lo anterior es posible al detallar cómo se está en términos de habilitación de funciones mentales y su uso en la realización de las actividades de la vida diaria.

Los tres calificadores de la Batería reconocen que la valoración de la funcionalidad que se hace con miras a la rehabilitación debe darse en el contexto de la estimulación de las funciones mentales en el ejercicio de los dominios y que aquella se da en el contexto de una relación de cuidado en la que las personas cuidadoras prestan su apoyo a las personas diagnosticadas con trastorno mental. La estimulación, entonces, es una actividad de doble vía y, al valorar la funcionalidad de la persona, se obtiene información acerca de cómo está llevando su vida en el desarrollo de las actividades de la vida diaria, así como qué ayudas adicionales necesita para que su proceso de rehabilitación sea adecuado. Es por esto que los instrumentos de la Batería logran tutorar el cuidado de las personas que se encuentran en procesos de rehabilitación en salud mental de base comunitaria.

Los registros basados en los calificadores descritos son recogidos en el proceso de valoración del funcionamiento mental, el cual se expresa en el tercer instrumento de la Batería: la matriz de evoluciones (ME). Esta matriz arroja los siguientes resultados: a) nivel del déficit del funcionamiento mental expresado en porcentaje, de acuerdo con las bases conceptuales de la CIFOMS; b) histórico semestral del nivel de déficit de funcionamiento mental; c) perfil de funcionalidad mental por eje de intervención (autocuida- 
do, convivencia, ocupación); d) perfil por calidad de la ejecución, y e) perfil según calidad de la ejecución por eje de intervención. Según estos datos, se logran los diagnósticos así: a) clasificación de la extensión del déficit (primer calificador); b) naturaleza del déficit (segundo calificador); c) perfil de desempeño menor logro; d) porcentaje de apoyo según la CIF-OMS, y e) diagnósticos enfermeros, con los que se construye el plan de cuidados y las intervenciones terapéuticas recomendadas, que proyectan los resultados esperados y los indicadores de medición articulados a la Batería, según la evidencia científica para los cuidados enfermeros, según la CIF-OMS y el NANDA-NIC-NOC. Toda esta información tiene la ventaja de estar expresada en los lenguajes estandarizados de la CIF-OMS y de las guías de diagnóstico e intervenciones del NANDA, NIC, NOC, lo que permite una mayor interoperatividad de los procesos de rehabilitación de base comunitaria. Otra de las virtudes de la ME es que, si bien emplea el lenguaje estandarizado de la CIF-OMS, también hace un énfasis considerable en el histórico de la evolución de la funcionalidad mental de la persona, en las relaciones que tienen los aspectos cognitivos, emotivos y ejecutivos, así como su aplicación en los tres ejes de intervención, el autocuidado, la convivencia y la ocupación, tanto a nivel global, como a nivel de los grupos de funciones mentales.

En conclusión, la Batería se distancia de la visión médica de la funcionalidad de la CIF-OMS cuando se trata de estimular y registrar la manera en que las personas en proceso de rehabilitación realizan su cotidianidad y participan en las actividades de su comunidad, pero rescata algunos elementos, como la escala de déficit, para ayudar a la interoperatividad de sistemas de atención y cuidado, que deben articularse para que pueda haber una rehabilitación exitosa. Este distanciamiento crítico es precisamente lo que permite que la Batería pueda a la vez guiar la estimulación en los procesos de rehabilitación y servir como herramienta de valoración de la funcionalidad que le habla a los diferentes profesionales involucrados en dicho proceso.

\section{Sección III. Conclusiones}

Este artículo se centró en presentar las relaciones entre la Batería de Estimulación y Valoración de la Funcionalidad Mental, construida por la organización Asociación Coometas Campesinas Saludarte y la Universidad Juan N. Corpas, así como la Clasificación Internacional de la Discapacidad y la Funcionalidad (CIF-OMS). Presentó, además, las reflexiones y críticas a la CIF-OMS que se produjeron durante el proceso de construcción de la Batería. A continuación, se listan las conclusiones más relevantes del artículo, con miras a contribuir a abrir un camino de exploración para lograr una comprensión de la funcionalidad mental, desde la rehabilitación de base comunitaria, que integre efectivamente la perspectiva de los cuidados de la enfermería.

1. La CIF-OMS, el principal referente para la clasificación de la discapacidad y la funcionalidad, aunque procura integrar el modelo social de la discapacidad, aún mantiene aspectos determinantes del modelo médico, los cuales deberían estar en coherencia con los principios de la rehabilitación de base comunitaria en salud mental. Un claro ejemplo es que los constructos y calificadores de esta mantienen una visión de la discapacidad como la desviación de la norma, perspectiva claramente capacitista y distanciada de la perspectiva desde la filosofía de la rehabilitación. En esta, el racero de la funcionalidad no es la comparación desde la mirada hegemónica de los cuerpos, sino la medida en que una persona puede llevar a cabo las actividades de la vida diaria y logra tener una vida con sentido, centrada en sí misma.

Esta reflexión trae preguntas acerca de qué tan adecuado resulta utilizar la CIF-OMS a ojos cerrados para guiar e informar los procesos de rehabilitación de base comunitaria en salud mental. La Batería de Estimulación y Valoración de la Funcionalidad Mental se construyó a partir del uso de ojos abiertos al lenguaje de la CIF-OMS, al integrar la perspectiva enfermera en aquellos aspectos que dicho lenguaje no permitía abordar. Algunos de estos son las diferencias sutiles entre habilitación, uso y uso exitoso de las funciones mentales, que requieren una atención a detalles que las taxonomías de la CIF-OMS no cubren. 
2. La adecuada comprensión de las funciones mentales es un aspecto fundamental para los procesos de rehabilitación en salud mental de base comunitaria y, sin embargo, las principales clasificaciones en salud mental no le dan un papel preponderante a explicar qué perspectivas adoptan para sus taxonomías. Esto no solo sucede en el caso de la CIF-OMS, sino también en el caso de la Clasificación Internacional de las Enfermedades, décima edición (CIE10). De hecho, fue solo hasta el 2013 que el Manual Diagnóstico y Estadístico de los Trastornos Mentales (DSM-V) incluyó una taxonomía y lo hizo únicamente como un complemento a la definición de trastornos muy específicos.

3. El reto de construir herramientas que se aproximen a la funcionalidad desde la articulación de las funciones mentales y las actividades de la vida diaria requiere que dichas herramientas cuenten con taxonomías de las funciones mentales que estén en consonancia con la filosofía y programas de rehabilitación, si lo que se busca es contribuir a los procesos de rehabilitación en salud mental de base comunitaria. Esto puede requerir el uso de taxonomías diferentes a las propuestas por las clasificaciones más conocidas (como la CIFOMS, CIE10 y DSM-V) o incluso proponer que estas clasificaciones realicen el trabajo adicional necesario. En ambos casos, integrar la perspectiva del trabajo de enfermería en salud mental resulta altamente relevante.

4. Es importante tomar con distancia crítica el enfoque biopsicosocial adoptado por la CIFOMS al construir instrumentos asociados a la rehabilitación basada en comunidad, en la medida en que aquellas afirmaciones que mantienen el modelo médico de la discapacidad pueden ir en contravía de los principios de esta. No obstante, en tanto el sistema de salud y protección social de Colombia aún sigue un modelo médico, resulta útil integrar algunos de esos aspectos para lograr los niveles de interoperatividad necesarios para sostener procesos de rehabilitación adecuados. Esta es una línea delgada, que la Batería ha logrado mantener, lo que da esperanza a otras herramientas para que busquen seguir este camino.

5. Finalmente, es importante resaltar que la Batería de Estimulación y Valoración de la Funcionalidad Mental, al ser un producto de un proceso de investigación crítico y riguroso desde un compromiso claro con la perspectiva de la rehabilitación en salud mental de base comunitaria, ha logrado responder a múltiples retos y necesidades insatisfechas que había en el contexto de la valoración funcionalidad mental. Esto se logró, principalmente, mediante la integración de los resultados del trabajo del personal de enfermería con las personas residentes del centro de rehabilitación en salud mental de base comunitaria A.SALUDARTE, y mediante la integración de marcos conceptuales de enfermería a la construcción de la Batería, como por ejemplo el manual de Mariño y Pabón (2011). Esto muestra que la perspectiva enfermera de la rehabilitación de base comunitaria puede aportar en enriquecer la comprensión de la funcionalidad y la discapacidad en salud mental. Así las cosas, se espera que esta conclusión permita abrir un camino de exploración para construir una comprensión de la funcionalidad mental que integre efectivamente la perspectiva de enfermería.

\section{Referencias Bibliográficas}

1. Al-Aidroos N., Said C.P., Turk-Browne N.B. (2012). Top-down attention switches coupling between low-level and high-level areas of human visual cortex. Proceedings of the National Academy of Sciences of the United States of America, 109(36), 14675-14680. https://doi.org/10.1073/ pnas.1202095109

2. American Psychiatric Association. (2013). Diagnostic and Statistical Manual of Mental Disorders. 5ta edición. American Psychiatric Association. 
3. Andrianopoulos, V., Gloeckl, R., Vogiatzis, I., Kenn, K. (2017). Cognitive impairment in COPD: Should cognitive evaluation be part of respiratory assessment? Breathe, 13, e1-e9. https://doi. org/10.1183/20734735.001417

4. Anthony, W.A. (1979). Principles of psychiatric rehabilitation. Pro-Ed.

5. Anthony, W.A. (1994). Characteristics of people with psychiatric disabilities that are predic tive of entry into the rehabilitation process and successful employment outcomes. Psychosocial Rehabilitation Journal, 17(3), 3-13. https://doi.org/10.1037/h0095578

6. Anthony, W.A., \& Jansen, M. (1984). Predicting the vocational capacity of the chronically mentally ill: Research \& policy implications. American Psychologist, 39, 537-544. https://doi.org/10.1037//0003-066x.39.5.537

7. Anthony, W.A., \& Liberman, R.P. (1986). The practice of psychiatric rehabilitation: Historical, conceptual, and research base. Schizophrenia Bulletin, 12, 542-559. https://doi.org/10.1093/schbul/12.4.542 Anthony, W.A., Cohen, M.R., \& Cohen, B.F. (1984). Psychiatric rehabilitation. In J.A. Talbot (Ed.), The chronic mental patient: Five years later (pp.137-157). Grune \& Stratton.

8. Anthony, W.A., Cohen, M.R., \& Vitalo, R. (1978). The measurement of rehabilitation outcome. Schizophrenia Bulletin, 4, 365-383.

9. Arns, P.G., \& Linney, J.A. (1995). Relating functional skills of severely mentally ill clients subjective and societal benefits. Psychiatric Services, 46(3), 260-265. https://doi.org/10.1176/ps.46.3.260 Babcock, H.A. (1930). An experiment in the measurement of mental deterioration. Archives of Psychology, 117, 105.

10. Bouttelet, D., Ferreira, N., Toyoko, S., Souza, M., de Mello B. (2008). Análisis de la producción científica de los encuentros de investigadores en salud mental y encuentro de especialistas en enfermería psiquiátrica. Rev. Latino-Am. Enfermagem, 16(3). http://dx.doi.org/10.1590/S010411692008000300022

11. Cohen, B., \& Anthony, W.A. (1984). Functional assessment in psychiatric rehabilitation. In A. Halpern \& M. Fuhrer (Eds.), Functional assessment in rehabilitation. Paul H. Brookes.

12. Cooke, K.Z., Fisher, A.G., Mayberry, W., Oakley, F. (2000). Differences in activities of daily living process skills of persons with and without Alzheimer's disease. OTJR: Occupation, Participation and Health, 20(2), 87-105. https://doi.org/10.1177/153944920002000201

13. Dahlstrom, W., Welsh, G., \& Dalstrom, L. (1972). An MMPI handbook: Clinical interpretation (Vol. 1). University of Minnesota Press.

14. Damasio, H., Damasio A.R. (1989). Lesion Analysis in Neuropsychology. Oxford University Press.

15. Derogatis, L.R. (1994). Symptom checklist-90-R (SCL-90-R) administration, scoring, \&procedures manual (3ra ed.). National Computer Systems.

16. Derogatis, L.R., Rickels, K., \& Rock, A. (1976). The SCL-90 and the MMPI: A step in the validation of a new self-report scale. British Journal of Psychiatry, 128, 280-289. https://doi.org/10.1192/ bjp.128.3.280 
17. Dion, G.L., \& Anthony, W.A. (1987). Research in psychiatric rehabilitation: A review of experimental and quasi-experimental studies. Rehabilitation Counseling Bulletin, 30, 177-203

18. Eisen, S.V., Dill, D.L., \& Grob, M.C. (1994). Reliability and validity of a brief patient-report instrument for psychiatric outcome evaluation. Hospital and Community Psychiatry, 45, 242-247. https://doi.org/10.1176/ps.45.3.242

19. Grossman, M., \& Irwin, D. (2016). The Mental Status Examination in Patients with Suspected Dementia. CONTINUUM: Lifelong Learning in Neurology, 22, 385-403. https://doi.org/10.1212/ CON.0000000000000298

20. Haffey W.J., Johnston M.V. (1990) A Functional Assessment System for Real-World Rehabilitation Outcomes. In Tupper D.E., Cicerone K.D. (Eds.), The Neuropsychology of Everyday Life: Assessment and Basic Competencies. Foundations of Neuropsychology (vol. 2). Springer, Boston, MA. https://doi.org/10.1007/978-1-4613-1503-2_5

21. Harvey, P. (2019). Domains of cognition and their assessment. Dialogues in Clinical Neuroscience, 21(3), 227-237. https://doi.org/10.31887/DCNS.2019.21.3/pharvey

22. Honigfeld, C., \& Klett, C. (1964). NOSIE: A new scale for measuring improvement in chronic schizophrenics. Newsletter of Research Psychology, 6, 22-23.

23. Lezak, M. D., Howieson, D. B. \& Loring, D. W. (2004) Neuropsychological Assessment 4. Oxford University Press.

24. López, M., \& Laviana, M. (2007). Rehabilitación, apoyo social y atención comunitaria a personas con trastorno mental grave: propuestas desde Andalucía. Revista de la Asociación Española de Neuropsiquiatría, 27(1), 187-223.

25. López-García P., Lesh, T.A., Salo, T., Barch, D.M., MacDonald, A., Gold, J.M., Ragland, J.D., Strauss, M., Silverstein, S.M., \& Carter, C.S. (2016). The neural circuitry supporting goal maintenance during cognitive control: a comparison of expectancy AX-CPT and dot probe expectancy paradigms. Cognitive, Affective \& Behavioral Neuroscience, 16(1), 164-175. https://doi.org/10.3758/ s13415-015-0384-1 Mariño, E. y Pabón, M. (2011) Manual valoración de funciones mentales. Universidad El Bosque, Facultad de Enfermería.

26. Mesulam, M.M. (Ed.). (2000). Principles of Behavioral and Cognitive Neurology (2da ed.). Oxford University Press.

27. NANDA Internacional. (2008) Diagnósticos Enfermeros. Definiciones y Clasificación. Madrid. https://biblioteca.ugr.es/pages/biblioteca_electronica/libros_enciclopedias_electronicos/nandaenfermeria

28. Organización Mundial de la Salud. (2001). Clasificación internacional del funcionamiento de la discapacidad y de la salud: CIF-OMS: versión abreviada. Ginebra: Organización Mundial de la Salud. https://apps.who.int/iris/handle/10665/43360 
29. Organización Panamericana de la Salud. (2015). Clasificación Estadística Internacional de Enfermedades y Problemas Relacionados con la Salud. Oficina Sanitaria Panamericana, Oficina Regional de la Organización Mundial de la Salud. https://www.paho.org/hq/dmdocuments/2016/2016 -cha-cie-10-10ma-revision.pdf

30. Schaber, P., Klein, T., Hanrahan, E., Vencil, P., Afatika, K., \&Burns, T. (2013). Using Cognitive-Functional Assessment to PredictSelf-Care Performance of Memory CareTenants. American Journal ofAlzheimer's Disease \& Other Dementias, 171-178. https://doi.org/10.1177/1533317512470206 